\title{
Electrostrictive laser-induced gratings for time-resolved observation of translational-rotational energy transfer in $\mathbf{H}_{2}$
}

\author{
W. Hubschmid • R. Bombach
}

Received: 20 August 2010 / Revised version: 18 November 2010 / Published online: 17 December 2010

(C) Springer-Verlag 2010

\begin{abstract}
Electrostrictive laser-induced gratings (LIG) have been formed in $\mathrm{H}_{2}$ of various pressures $(0.5 \ldots 5$ bar $)$ and with frequencies of the Brillouin modes (sound waves) of about $14 \mathrm{MHz}$. Under these conditions, the rotational degrees of freedom can only partially follow the temperature variation from the acoustic oscillation. A model is given, which completes the linearized fluid dynamical equations with equations for the rotational state relaxation, separately for the average relaxation of the states for Ortho- $\mathrm{H}_{2}$ and Para- $\mathrm{H}_{2}$. Using such a model, the dispersion of sound frequency is reproduced with an accuracy of a few percent. However, the total dissipation of sound and the observed strong alternation in peak heights in the temporal evolution of the LIG intensity is only approximately predicted by the model.
\end{abstract}

\section{Introduction}

Absorption of ultrasound in pure monatomic gases, in the fluid dynamic regime, originates from the so-called classical dissipation processes, i.e., heat conduction and shear viscosity. In polyatomic gases an enhanced sound absorption, beyond classical absorption, is observed; it is attributed to intramolecular energy transfer [1, 2]. Actually, in frequency domains where translational and internal degrees of freedom, viz. rotations or vibrations, are not in equilibrium with each other, the sound absorption due to intramolecular energy transfer may be larger by orders of magnitudes than the

W. Hubschmid $(\bowtie) \cdot$ R. Bombach

Paul Scherrer Institut, 5232 Villigen PSI, Switzerland

e-mail: walter.hubschmid@psi.ch

Fax: +41-56-3102199 sound absorption caused by the classical processes of dissipation. This has been noted since long, see Refs. $[1,2]$ or Kneser [3] and references cited therein.

In the usual approaches to measure (ultra)sound absorption, sound is generated by an actuator and, after some propagation in the medium, detected by a sensor, or the sound absorption is obtained by applying interferometric techniques. Knowledge of (frequency-dependent) parameters of sound absorption-besides the classical parameters that are measurable in static experiments - can be obtained in these techniques by varying frequency and/or pressure of the medium.

In contrast to this, in the time-resolved technique using electrostrictive laser-induced gratings (LIG) [4-7], sound is generated simultaneously in the whole overlap volume of the laser beams. In this volume, two sound waves (Brillouin modes), which counterpropagate in normal direction to the layers of the electric field strength and build up a standing sound wave, are generated. Moreover, besides the Brillouin modes, a Rayleigh mode (density wave at constant pressure) as well is excited, as was shown in Ref. [4]. Altogether, these modes build up a transient optical grating in the medium that can be read out by a cw-laser beam, so that the temporal evolution of the grating can be recorded. The intensity of the diffracted probe beam is called LIG signal intensity. Preferably the medium is transparent for the read-out laser.

In the time-resolved LIG technique, nonclassical parameters of sound absorption can, with limited accuracy at the stage of investigation as presented in this paper, be determined in a single experiment. To investigate such a possibility of measurements and to obtain the phenomenology of the temporal evolutions of LIG in dispersive domains are the main goals of this article. The investigations were performed on $\mathrm{H}_{2}$ of various pressures $(0.5 \ldots 5$ bar $)$, with frequencies for the sound generated of about $14 \mathrm{MHz}$. This corresponds to the dispersive domain of translational-rotational energy transfer. 


\section{Electrostrictive LIG experiment for the observation of sound absorption}

For the experiments described in this paper, laser beams for the formation of the LIG are unfocused. This, on the one hand, reduces the spatial resolution but, on the other hand, enlarges strongly the propagation time of the sound wave out of the observation volume. Thus, in the time evolution of the LIG signal intensity, attenuation from dissipation of the sound waves can better be distinguished from attenuation by their propagation out of the observation volume.

The setup used in our LIG experiments to determine the sound absorption of $\mathrm{H}_{2}$ (or of other gases) is depicted to scale in Fig. 1. A Quantel YG581C multimode Nd:YAG laser delivered the LIG forming beams at $1064 \mathrm{~nm}$. The pulse energy per beam was approximately $450 \mathrm{~mJ}$ with pulse duration of $14 \mathrm{~ns}$ (long-pulse option). A template reduced the beam width to $4 \mathrm{~mm}$. The precise angle of the pump beams $\left(0.639^{\circ}\right)$ was derived from the observed LIG signal oscillation frequency (respectively the period $T$ ) recorded

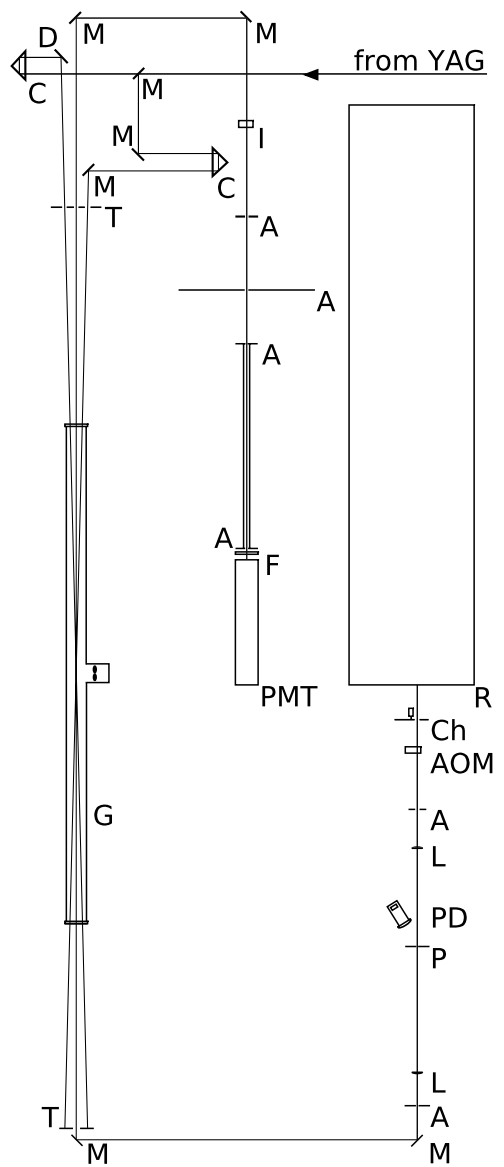

Fig. 1 Experimental setup. A: apertures. AOM: acousto-optic modulator. $C$ : corner cube prisms. $C h$ : chopper. $D$ : dichroic mirror (see text). $G$ : gas cell. $F$ : infrared blocking filter. $I$ : interference filter. $L$ : lenses. $M$ : mirrors. $P$ : pinhole. $P D$ : photodiode. $P M T$ : photomultiplier. $R$ : argon ion readout laser. $T$ : cardboard beam templates in $\mathrm{Ar}$ and $\mathrm{N}_{2}$, and from the known sound velocity $v$ of these gases, which leads to the value for the distance $\Lambda$ between the grating layers.

The 514-nm readout beam was provided by a Spectra Physics 2060-5S Beamlok argon ion laser. Focusing optics kept the read-out beam diameter below $1 \mathrm{~mm}$. The output power was adjusted between $125 \mathrm{~mW}$ and $1 \mathrm{~W}$ to accommodate with the dynamic range of the detector. The wavelength had to be chosen off $532 \mathrm{~nm}$ because there is some second harmonic generation in all optical components exposed to the 1064-nm radiation of the pump beam YAG laser.

Background load to the detector PMT was reduced in three steps. First, a mechanical chopper wheel synchronized with the laser pulses cut down the averaged intensity by a factor of 100. Subsequently an acousto-optic modulator sliced out a pulse of 10-20 $\mu$ s, depending on experiment. Finally the beam was sent through a spatial filter consisting of an $f=200-\mathrm{mm}$ lens, a $100-\mu \mathrm{m}$ pinhole, and an $f=300-\mathrm{mm}$ lens. The beam diffracted at the LIG passes several apertures, a 514-nm interference filter, and a Schott KG3 infrared blocking filter. In case of small beam angles, the readout beam passes first the dichroic mirror $\mathrm{D}$. The intensity of the signal beam was detected with a Hamamatsu R3234-01 high-speed, low-afterpulse photomultiplier tube (rise time $1.3 \mathrm{~ns}$ ). A Tektronix TDS 744A digital storage oscilloscope (500 MHz, $2 \mathrm{GS} / \mathrm{s})$ served as recorder and signal averager. Typically 400 to 800 , if needed occasionally up to 4000 , signal pulses have been averaged. The gas cell consists of standard 40-mm Klein flange components which have been equipped with dual-wavelength anti-reflection coated 2" quartz glass windows at the ends. As beam overlap within the windows creates strong parasitic signals, the length of the cell had to be changed depending on the beam angle used. The maximum cell length used was $1.3 \mathrm{~m}$. The pressure was monitored with a MKS Baratron membrane manometer.

\section{Theory of LIG formation and decay}

The time-dependent LIG signal intensity is proportional to the square of the time-dependent variation of the refractive index in the grating, which is formed in the overlap volume of the laser beams [8]. The time-dependent variation of the refractive index itself is proportional to the variation $\rho^{\prime}=\rho-\rho_{0}$ of the local mass density around its temporally averaged value $\rho_{0}$. Other variations of physical quantities, which contribute to a variation of the refractive index, as variation of the populations of the molecular states, and variation of the temperature, are assumed to be not of relevance in our experiments, and they are therefore not considered here. The geometrical factors, like the extension of the grating, are not calculated either; they are included in an overall factor. 
The temporal variation of the mass density in the grating volume can be calculated by means of the linearized equations of fluid dynamics for momentum and energy transport. Variables in these equations are $\rho^{\prime}=\rho-\rho_{0}$ and the variation $T^{\prime}=T-T_{0}$ of the local temperature $T$ around the average value $T_{0}$. With a term added on the right-hand side (RHS) of the momentum equation, which represents the electrostrictive force, the equations have the following form [9]:

$$
\begin{aligned}
& \frac{\partial^{2} \rho^{\prime}(t, \vec{x})}{\partial t^{2}}-\frac{\mathbf{v}^{2}}{\gamma} \Delta \rho^{\prime}(t, \vec{x})-\frac{\mathbf{v}^{2} \beta_{p} \rho_{0}}{\gamma} \Delta T^{\prime}(t, \vec{x}) \\
& -\frac{\eta}{\rho_{0}} \frac{\partial}{\partial t} \Delta \rho^{\prime}(t, \vec{x})=-\frac{\varepsilon_{0} \gamma_{e}}{2} \Delta \mathbf{E}^{2}, \\
& \rho_{0} c_{\mathrm{v}} \frac{\partial T^{\prime}(t, \vec{x})}{\partial t}-\frac{\left(c_{p}-c_{\mathrm{V}}\right)}{\beta_{p}} \frac{\partial \rho^{\prime}(t, \vec{x})}{\partial t}-\kappa \Delta T^{\prime}(t, \vec{x})=0 .
\end{aligned}
$$

Symbols used in (1), (2) are: $v$ is the adiabatic sound velocity, $c_{p}$ is the heat capacity at constant pressure, $c_{\mathrm{v}}$ is the heat capacity at constant volume, $\gamma$ is the ratio $c_{p} / c_{\mathrm{v}}, \eta$ is the total viscosity, i.e., $\eta=(4 / 3) \eta_{s}+\eta_{b}$, with the shear viscosity $\eta_{s}$ and the bulk viscosity $\eta_{b}$, which enters here as a free parameter. Furthermore, $\Delta$ is the Laplacian, $\beta_{p}$ is the thermal expansion coefficient at constant pressure, $\kappa$ is the thermal conductivity, $\gamma_{e}=\rho(\partial \varepsilon / \partial \rho)$ is the electrostrictive constant, and $n$ is the refractive index.

The electric field $\mathbf{E}$, which results from two beams of a laser, intersecting at an angle $\theta$, is approximated in (1) by a $\delta$-function in time. It is considered to be infinitely spatially extended in the formal representation of (1), in order to still be able to solve analytically the equations of motion. Finite extension (in transverse direction) is only considered later in data analysis through a finite propagation time of the sound waves out of the observation volume. The electric field intensity $\mathbf{E}^{2}$ has a part, which is independent on the spatial position, and a spatially dependent part, which contributes to the term of the RHS of (1). This term is denoted by $\mathbf{E}_{\mathrm{var}}^{2}$; its strength $I$ is defined according to

$\mathbf{E}_{\mathrm{var}}^{2}=I \cos q x \delta\left(t-t_{0}\right)$,

where $q$ is the modulus of the grating vector, $q=2 \pi / \Lambda$, with the distance $\Lambda$ between grating layers. (The notation in (3) is slightly different from the one used in the earlier article [6].) $\Lambda$ is related to the wavelength $\lambda$ of the grating forming beams by $\Lambda=\lambda /(2 \sin (\theta / 2))$. In general, in the RHS of (2), also sources of heat, e.g., from absorption of light of the LIG formation beams, may be introduced. However, in our experiments, light absorption is not relevant. Also, dissipative processes as a source of heat are not considered here, as it is not of linear order in $\rho^{\prime}$ and $T^{\prime}$ [9]. (We checked by an estimate that such contribution actually can be neglected.)

It was shown in Ref. [4] that experimental data of the time-resolved LIG intensity with $\mathrm{N}_{2}$ and Ar as media, at frequencies of about $30 \mathrm{MHz}$, can well be modeled by (1), (2). As there is no internal molecular energy transfer (Ar), or there is, in good approximation, thermal equilibrium between translational states and internal energy states $\left(\mathrm{N}_{2}\right.$ : rotational states), it is justified to insert into the equations the static values for the specific heat capacities and the sound velocity. A value for the bulk viscosity is determined in the case of $\mathrm{N}_{2}$, which takes into account that the observed sound dissipation is larger than from thermal conduction and shear viscosity alone. It was shown furthermore in Ref. [4] that the initial-value problem posed by (1), (2) is solved by a superposition of two counterpropagating sound waves (Brillouin modes) and, with lower amplitude, a spatially stationary density variation (Rayleigh mode). This mode is responsible for the experimentally observed odd-even behavior of the peaks of the LIG intensity; i.e., the odd-numbered peaks have a somewhat enhanced intensity compared with the even-numbered peaks.

\section{Periodic translational-rotational energy transfer in $\mathbf{H}_{2}$}

When there is slow intramolecular heat transfer between translational and internal degrees of freedom, as is the case for $\mathrm{H}_{2}$ in the domain of a few MHz/bar, a somewhat different situation arises. Under these conditions, the rotational degrees of freedom can only partially follow the temperature variation from the acoustic oscillation. In consequence, specific heat capacities-if variations of translational temperatures are considered - are smaller, and the sound velocity, which is proportional to the square root of the ratio $\gamma$ of heat capacities, is larger than in the low-frequency limit.

In order to describe such partial thermal equilibrium, besides the temperature $T$ of the translational motion, an internal temperature $T_{\text {int }}$ of the partially excited internal degrees of freedom is introduced [1]. In extension, temperatures $T_{\mathrm{int}, i}(t)$ for each state of the internal degrees of freedom may be considered. In modeling the dynamics, the linearized fluid dynamic equations (1), (2) have thus to be supplemented with equations which describe the relaxation of the internal temperatures, i.e., in case of $\mathrm{H}_{2}$, the rotational temperatures. In this work, in order to simplify the data analysis and to avoid too much arbitrariness in determining parameters, we model the rotational state relaxation by including only two processes, each with a relaxation constant. This is thought to refer to the relaxation in Ortho- $\mathrm{H}_{2}$ and $\mathrm{Para}-\mathrm{H}_{2}$, respectively. Thus, we introduce two rotational temperatures $T_{\mathrm{int}, i}, i=1,2$.

To describe relaxation, commonly the assumption is made that the temporal derivative of the relaxing quantity is proportional to its difference with the corresponding quantity for thermal equilibrium. Thus, in our case,

$$
\dot{T}_{\mathrm{int}, i}=-\lambda_{i}\left(T_{\mathrm{int}, i}-T\right) \text {. }
$$


From (4) it follows that the variations of the internal temperatures are time delayed with respect to variations of the translational temperature. In case of a periodic variation of the translational temperature, amplitudes of the oscillations of the internal temperatures are reduced, and the oscillations are phase-shifted with respect to the translational temperature. In consequence, pressure and mass density oscillate at different phases. This gives rise to an additional internal friction, i.e., to a nonvanishing value of the bulk viscosity. Actually, neglecting heat transfer in zeroth order, the pressure oscillates with the phase of the translational temperature, whereas the density oscillates with the phase of the total internal energy, i.e., the sum of the translational and the intramolecular energy.

Furthermore, when relaxation is present in the system to be described, the heat transport equation (see (2)) has to be adapted. The internal energy term is split into separate terms for translational energy and for intramolecular energy with the specific heat capacities $c_{\mathrm{v}, \text { tr }}$ and, from counting degrees of freedom, $c_{\mathrm{v}, \text { int }}=2 / 3 c_{\mathrm{v}, \mathrm{tr}}$. Taking into account that the densities $\rho_{0,1}$ and $\rho_{0,2}$ for Ortho- $\mathrm{H}_{2}$ and Para- $\mathrm{H}_{2}$, respectively, are $\rho_{0,1}=3 / 4 \rho_{0}$ and $\rho_{0,2}=1 / 4 \rho_{0}$, one obtains thus the modified energy transport equation

$$
\begin{gathered}
\rho_{0} c_{\mathrm{v}, \operatorname{tr}}\left(\frac{\partial T^{\prime}(t, \vec{x})}{\partial t}+\frac{1}{2} \frac{\partial T_{\mathrm{int}, 1}^{\prime}(t, \vec{x})}{\partial t}+\frac{1}{6} \frac{\partial T_{\mathrm{int}, 2}^{\prime}(t, \vec{x})}{\partial t}\right) \\
-\frac{c_{p}-c_{\mathrm{V}}}{\beta_{p}} \frac{\partial \rho^{\prime}(t, \vec{x})}{\partial t}-\kappa \Delta T^{\prime}(t, \vec{x})=0 .
\end{gathered}
$$

Primed quantities in (5) are used for the deviations of quantities from their temporal average. Equations (1), (4), (5) form the modified system of differential equations, which is proposed in case of nonequilibrium between translational and internal degrees of freedom.

\section{Solution of the modified linearized fluid dynamic equations}

In a first step of solving the coupled linearized fluid dynamic equations, the space dependence of $\rho^{\prime}(t, \vec{x}), T^{\prime}(t, \vec{x})$, and $T_{\mathrm{int}, i}^{\prime}(t, \vec{x})$ in (1), (4), (5) is split off. This is done under the assumption noted above that grating layers are infinitely extended in the $y-z$ plane (symmetry axis of irradiating laser beams in direction of $y$-axis). Hence,

$$
\begin{aligned}
& \rho^{\prime}(t, \vec{x})=\cos q x \rho^{\prime}(t), \quad T^{\prime}(t, \vec{x})=\cos q x T^{\prime}(t), \\
& T_{\mathrm{int}, i}^{\prime}(t, \vec{x})=\cos q x T_{\mathrm{int} . i}^{\prime}(t) .
\end{aligned}
$$

In the next step, the functions $\rho^{\prime}(t), T^{\prime}(t)$, and $T_{\mathrm{int}, i}^{\prime}(t)$ are Fourier transformed according to

$$
\tilde{f}(\Omega)=\int_{-\infty}^{+\infty} f(t) \exp (-i \Omega t) d t .
$$

The Fourier transforms (FT) of (4) are given by

$\left(i \Omega+\lambda_{i}\right) \tilde{T}_{\mathrm{int}, i}=\lambda_{i} \tilde{T}$.

Relations (7) are inserted into the equation for the FT of (5). The FT $\tilde{\rho}(\Omega)$ and $\tilde{T}(\Omega)$ obey thus the following equations, where $\tau_{\mathrm{i}} \equiv 1 / \lambda_{i}$ :

$$
\begin{aligned}
& \Omega^{2} \tilde{\rho}-\frac{q^{2} v^{2}}{\gamma} \tilde{\rho}-\frac{\beta_{p} \rho_{0} q^{2} v^{2}}{\gamma} \tilde{T}-i \Omega \frac{\eta q^{2}}{\rho_{0}} \tilde{\rho} \\
& \quad=-\frac{1}{2} q^{2} \gamma_{e} \varepsilon_{0} I \exp \left(-i \Omega t_{0}\right), \\
& {\left[i \Omega \rho_{0} c_{\mathrm{v}, \operatorname{tr}}\left(1+\frac{1}{2\left(1+i \Omega \tau_{1}\right)}+\frac{1}{6\left(1+i \Omega \tau_{2}\right)}\right)+\kappa q^{2}\right] \tilde{T}} \\
& \quad=i \Omega \frac{c_{p}-c_{v}}{\beta_{p}} \tilde{\rho} .
\end{aligned}
$$

Substitution of $\tilde{T}(\Omega)$ from expression (9) into (8) and the inverse Fourier transform yields

$$
\begin{aligned}
\rho^{\prime}(t)= & -\frac{1}{4 \pi} q^{2} \gamma_{e} \varepsilon_{0} I \\
& \times \int_{-\infty}^{+\infty} \frac{\exp \left[i \Omega\left(t-t_{0}\right)\right]}{\left(\Omega^{2}-i h \Omega-k\right)-\frac{k l \Omega}{f(\Omega)-i m}} d \Omega
\end{aligned}
$$

with the notation

$f(\Omega)=\Omega\left(1+\frac{1}{2\left(1+i \Omega \tau_{1}\right)}+\frac{1}{6\left(1+i \Omega \tau_{2}\right)}\right)$,

$h=\frac{\eta q^{2}}{\rho_{0}}, \quad k=\frac{q^{2} v^{2}}{\gamma}$,

$l=\frac{c_{p}-c_{v}}{c_{\mathrm{v}, \mathrm{tr}}}, \quad m=\frac{\kappa q^{2}}{\rho_{0} c_{\mathrm{v}, \mathrm{tr}}}$.

The integral (10) can be calculated by applying the residuum technique: the integral path is closed for $t<t_{0}$ with a half circle in the lower half-plane and for $t>t_{0}$ in the upper halfplane. The poles of the integrand are all in the upper half plane; the general form for the roots of the denominator in the integrand is

$\Omega_{1}=\varpi+i \beta_{1}, \quad \Omega_{2}=-\varpi+i \beta_{1}$,

$\Omega_{3}=i \beta_{2}, \quad \Omega_{4}=i \beta_{3}, \quad \Omega_{5}=i \beta_{4}$,

where $\varpi, \beta_{1}, \beta_{2}, \beta_{3}, \beta_{4} \in \mathfrak{R}^{+} . \Omega_{1}$ and $\Omega_{2}$ are, up to the sign, the complex frequencies of the Brillouin modes, and $\Omega_{3} \ldots \Omega_{5}$ are, up to the sign, the decay constants in the three contributions to the Rayleigh mode, which decrease exponentially in time.

The residues are given by

$$
\begin{aligned}
\operatorname{Res}_{i} & =\lim _{\Omega \rightarrow \Omega_{i}} \frac{\left(\Omega-\Omega_{i}\right) \exp \left[i \Omega_{i}\left(t-t_{0}\right)\right]}{F(\Omega)} \\
& \equiv \operatorname{Res}_{i, \text { const }} \times \exp \left[i \Omega_{i}\left(t-t_{0}\right)\right]
\end{aligned}
$$


with the notation

$$
F(\Omega) \equiv \Omega^{2}-i h \Omega-k-\frac{k l \Omega}{f(\Omega)-i m} .
$$

Summing over the residues $\operatorname{Res}_{i}$ of the poles of the integrand, $i, i=1, \ldots, 4$, one obtains an expression of the form

$$
\begin{aligned}
\rho^{\prime}(t) \propto & \Theta\left(t-t_{0}\right) \times\left\{\operatorname { e x p } [ - \beta _ { 1 } ( t - t _ { 0 } ) ] \left\{\sin \left[\varpi\left(t-t_{0}\right)\right]\right.\right. \\
& \left.-\delta_{1} \cos \left[\varpi\left(t-t_{0}\right)\right]\right\}+\delta_{2} \exp \left[-\beta_{2}\left(t-t_{0}\right)\right] \\
& \left.+\delta_{3} \exp \left[-\beta_{3}\left(t-t_{0}\right)\right]+\delta_{4} \exp \left[-\beta_{4}\left(t-t_{0}\right)\right]\right\}
\end{aligned}
$$

for the temporal variation of the mass density. The quantities $\delta_{i}$ in (15), $i=1, \ldots, 4$, depend on the values of the timeindependent factors $\operatorname{Res}_{i, \text { const }}$ of the residua, as defined in the second equation of (13), and $\Theta\left(t-t_{0}\right)$ is the Heavisidefunction $\left(=1, t-t_{0}>0 ;=0, t-t_{0}<0\right)$.

\section{Experimental results and comparison with model}

The LIG experiments in $\mathrm{H}_{2}$ have been performed at pressures $p=0.5$ bar, 1 bar, 2 bar, and 5 bar. Figures $2 \mathrm{a}$ to $2 \mathrm{~d}$ show the respective results. They reveal an odd-even behavior of peak intensities, which is stronger for the lower pressures. A "generic" fit to the experimental curves reveals that they can rather well be reproduced by two contributions oscillating at angular frequencies $2 \varpi$ and $\varpi$, respectively, and both decaying exponentially. A closer look reveals then that the $\varpi$-term shows some deviation from a purely exponential decay. Such behavior would indeed be given by a function as derived in (15). However, as is shown below in detail, with the quantities $\Omega_{i}$ and $\delta_{i}$ determined from the solution of (1), (4), (5), the deviation from a purely exponential decay of the $\varpi$-term is by far too strong. In addition, the various terms that contribute to an oscillation of the LIG intensity with the angular frequency $\varpi$ do sum up only very approximately to the amount demanded by the experimental data.

Comparison of the model and experimental data was done in the following way: To take into account for the finite duration of the laser pulse and the finite detector response time, model curves were convoluted by a Gaussian with an FWHM of 14 ns. To take into account the propagation of the sound waves out of the observation volume, an overall envelope $H=\exp \left(-t^{2} / \sigma^{2}\right)$ of Gaussian shape was applied to the simulated density variation. From the data on sound velocity and the size of the pump beams, the width parameter $\sigma=2 \mu$ s seemed appropriate; such input exactly matches the value found from a best fit to the experimental data. (Neglecting this decay mechanism leads to an increase of the derived value of $\beta_{1}$ of $1.3 \%$ in the 0.5 -bar case. The influence is more pronounced when longer decay times are present, i.e., in the 5-bar case, but remains clearly below $1 \%$ when the corresponding decay time is changed by $10 \%$.)
In a third step, the various quantities in the model were determined. The ratio $l$ of specific heat capacities is $l=2 / 3$. For the intersecting angle $\theta=0.639^{\circ}$ between the laser beams, one obtains $q=65.87 \times 10^{3} \mathrm{~m}^{-1}$. Values for $k, m$, and the density $\rho_{0}$ are determined from tables [10]. Relaxation times and bulk viscosity are thus the only unknown quantities in (10), (11). In the next step, routines of Maple were used to determine the roots of the denominator of the integrand on the RHS of (10) and the corresponding residua. In particular the function "eval", with passing to the limit as $\Omega \rightarrow \Omega_{i}$, was used for the determination of residua. To be safe on the accuracy of the limit procedure, calculations were done with 40 digits.

The roots depend on values for $\tau_{i}$ and $\eta_{b}$ that are inserted in the expressions to be evaluated. To proceed here, the following general properties of the model are observed:

1. The angular frequency $\varpi$ depends strongly on $\tau_{i}$ but only very weakly on $\eta_{b}$. ( $\eta_{b}$ is for each single pressure assumed to be a frequency independent quantity.)

2. At the pressure $p=0.5$ bar, decay of the leading amplitude (dissipation constant $\beta_{1}$ ) is somewhat too strong even when inserting a vanishing value for $\eta_{b}$. For the higher pressures, some deficit in the modeled values of $\beta_{1}$ occurs, which may be attributed to bulk viscosity. However, as experimental data do not fit accurately with the model, we renounce on determining values for $\eta_{b}$ and insert in general a value $\eta_{b}=0.25 \eta_{s}$ in the model. This value compares about with values of the bulk viscosity determined for other gases beyond a dispersive domain [4]. At the pressure $p=0.5$ bar, the somewhat arbitrarily chosen value for $\eta_{b}$ contributes with $2.6 \%$ to the dissipation constant $\beta_{1}$; such value for $\eta_{b}$ contributes also little to $\beta_{1}$ for the other pressures.

3. Various pairs of $\tau_{1}$ and $\tau_{2}$ can reproduce the experimentally observed angular frequency $\varpi$. For the pressure $p=0.5$ bar, if $\tau_{1}=\tau_{2}$ is assumed, one obtains $\tau_{i}=26.2 \mathrm{~ns}$. This, however, leads at the higher pressures to relatively large deviations between the experimental and the theoretical value of $\varpi$. In order to obtain also here approximately correct values for the observed dispersion, i.e., the variation of the frequency with a variation of the pressure, we choose $\tau_{1}=24 \mathrm{~ns}$ and $\tau_{2}=80 \mathrm{~ns}$ for $p=0.5$ bar.

In Table 1, dependent on the pressure, the experimentally determined values for the angular frequency $\varpi$ and for the absorption coefficient $\beta_{1}$, and as well their values determined with the procedure described above, are noted. Thereby, it was taken into account that the relaxation times scale inversely with pressure. Also, the experimentally determined values for the sound velocity $v$, which is approximately given (see Ref. [5]) by $v=\varpi / q=\Lambda v$, and for the 
Fig. 2 Comparison of experiment and model for the temporal evolution of LIG intensity. (a) $p=0.5$ bar.

(b) $p=1$ bar. (c) $p=2$ bar.

(d) $p=5$ bar. The temperature was $295 \mathrm{~K}$. The frequencies in the modeled curves were adapted to their experimentally observed values. Solid lines: experimental data; dashed lines: fit as described in text
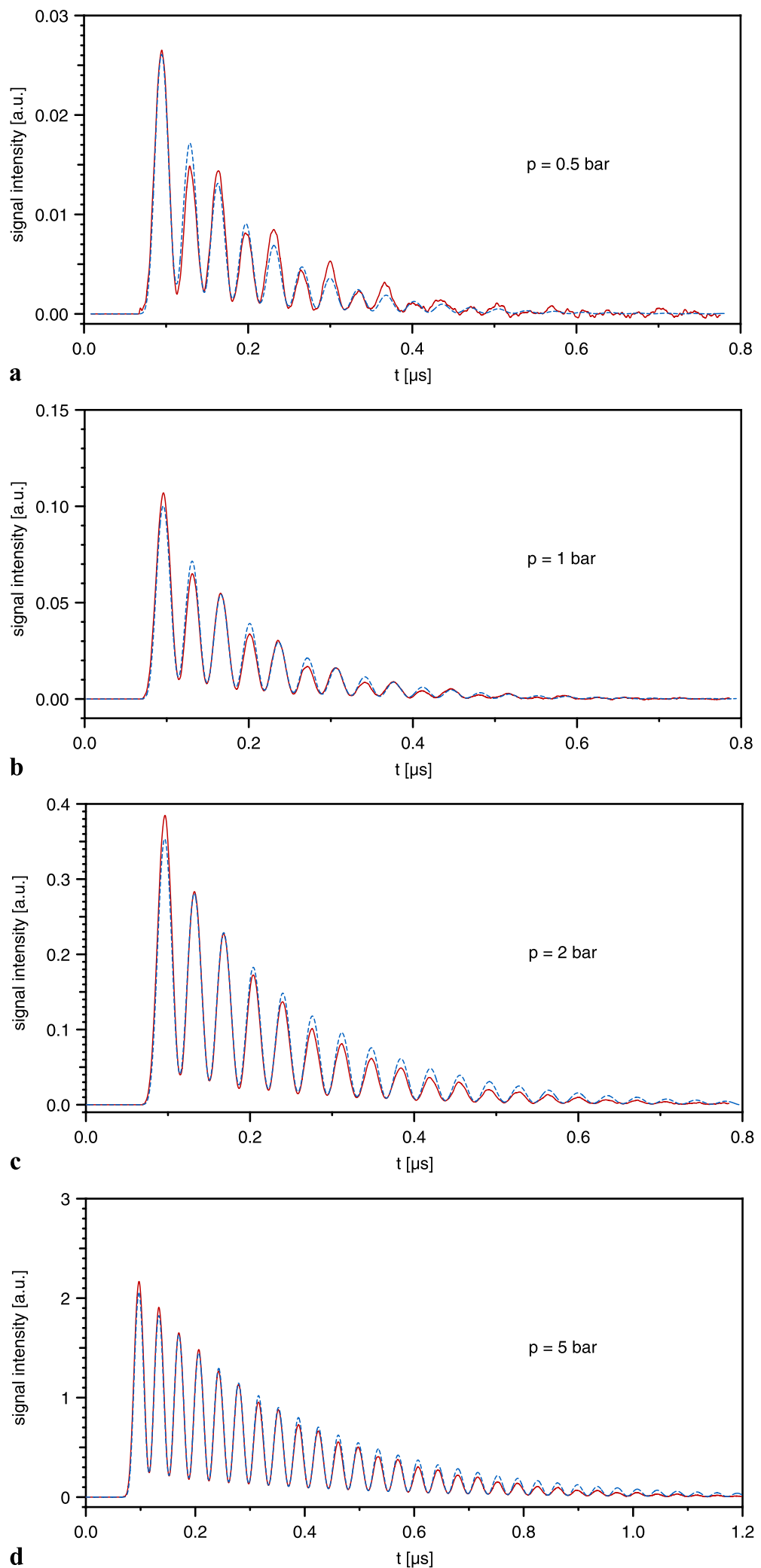

absorption coefficient $\mu \equiv \beta_{1} / v$ are noted in Table 1. Experimental values for $\varpi$ correspond to frequencies between $14.62 \mathrm{MHz}$ ( $p=0.5$ bar) and $13.74 \mathrm{MHz}$ ( $p=5$ bar).
The experimental and modeled values of $\varpi$ differ, relative to the deviations from the frequency that would arise for pressures close to 0 bar, by a few percent. (For 2 bar, 
Table 1 Experimental and modeled values for the angular frequency $\varpi$ of sound and the sound absorption coefficient $\beta_{1}$ at various ratios of $v_{\exp } / p$. In addition, the experimental values of the sound velocity $v$ and the absorption coefficient $\mu_{\exp } \equiv \beta_{1, \exp } / \nu_{\exp }$ are noted. The temperature was $295 \mathrm{~K}$

\begin{tabular}{lllll}
\hline$p$ [bar $]$ & 0.5 & 1 & 2 & 5 \\
$v_{\exp } / p[\mathrm{MHz} / \mathrm{bar}]$ & 29.24 & 14.26 & 6.97 & 2.747 \\
$\varpi_{\exp }\left[10^{6} \mathrm{~s}^{-1}\right]$ & 91.87 & 89.57 & 87.62 & 86.30 \\
$\varpi_{\bmod }\left[10^{6} \mathrm{~s}^{-1}\right]$ & 91.87 & 89.44 & 87.67 & 86.63 \\
$\beta_{1, \exp }\left[10^{6} \mathrm{~s}^{-1}\right]$ & 4.23 & 4.54 & 3.43 & 1.70 \\
$\beta_{1, \bmod }\left[10^{6} \mathrm{~s}^{-1}\right]$ & 4.67 & 4.25 & 2.95 & 1.53 \\
$v_{\exp }\left[\mathrm{m} \mathrm{s}^{-1}\right]$ & 1395 & 1360 & 1330 & 1310 \\
$\mu_{\exp }$ & 0.289 & 0.318 & 0.234 & 0.123 \\
\hline
\end{tabular}

the relative deviation is less than $1 \%$.) The values for $\mu$ are generally about $10 \%$ lower than the values given by Petralia [11]. The comparison of the experimental data for the temporal evolution of the LIG intensity with the modeled data is depicted in Figs. 2a to 2d. In order to facilitate comparison between experiment and model, the frequencies in the model data were set equal to the experimentally determined frequencies.

\section{Conclusions}

We showed in the present paper that the technique of electrostrictive LIG is well suited to investigate dispersion and absorption of sound in gaseous media. As LIG is a timeresolved technique, experimental data contain more information on these processes than the standard techniques. This may especially be useful for frequency domains where translational and internal degrees of freedom are not in thermal equilibrium with each other. Under such conditions, a periodic heat transfer between translational and intramolecular energy occurs, which changes the velocity and the absorption of sound. Conversely, from determining macroscopic parameters, such as sound velocity, sound absorption, and related quantities, data on the energy transfer between the different degrees of freedom can be inferred.

The experiments described in this paper were performed in $\mathrm{H}_{2}$ at pressures between 0.5 and 5 bar. The sound frequency was about $14 \mathrm{MHz}$. In this domain, translational and rotational degrees of freedom are not in equilibrium with respect to each other. Especially at the lower pressures, we observed that odd-numbered peaks in the temporal evolution of the LIG intensity are strongly enhanced compared with the even-numbered peaks. This may be attributed to the formation of a Rayleigh mode (density variation at constant pressure) of large amplitude in such LIG.
To model the observed behavior, the linearized fluid dynamical equations were completed with equations for the rotational state relaxation, separately for the average relaxation of the states for Ortho- $\mathrm{H}_{2}$ and Para- $\mathrm{H}_{2}$. The two relaxation constants, in a not fully unique procedure, were determined in such way that the observed sound velocities at the various pressures are approximately reproduced.

The model proposed does not reflect the whole phenomenology that is observed. There is approximate agreement between model and data for the observed dispersion: The relative deviations of the calculated sound velocity (with respect to the difference from its theoretical value at pressures close to 0 bar) from the experimentally observed values are reproduced within a few percent. The absorption of sound in dependence of the pressure is approximately reproduced, too. Though, at the smallest pressure $(0.5 \mathrm{bar})$, predicted absorption is somewhat larger than observed, even when the bulk viscosity is set equal to zero. In consequence, at the higher pressures, where the predicted absorption is smaller than what is observed, it is hardly justified to extract values for the bulk viscosity from the data.

The performance of the model is worst with respect to the general form of the temporal evolution of the LIG intensity: The enhancement of the odd-numbered peaks in the temporal evolution of the LIG intensity, compared with the evennumbered peaks, is predicted much smaller by the model than what is observed. Including additional equations, which describe the relaxation of some single rotational states, does not essentially improve the situation. Thus, we have to consider the model described here as a first attempt to theoretically explain experimental data on the LIG formation in a dispersive domain where translational and intramolecular degrees of freedom are not in equilibrium with each other.

\section{References}

1. K.F. Herzfeld, T.A. Litovitz, Absorption and Dispersion of Ultrasonic Waves (Academic Press, New York, 1959)

2. A.B. Bhatia, Ultrasonic Absorption (Clarendon, Oxford, 1967)

3. H.O. Kneser, in Handbuch der Physik, vol. XI/1 (Springer, Berlin, 1961)

4. W. Hubschmid, B. Hemmerling, A. Stampanoni-Panariello, J. Opt. Soc. Am. B 12, 1850 (1995)

5. W. Hubschmid, B. Hemmerling, A. Stampanoni-Panariello, Appl. Phys. B 62, 103 (1996)

6. A. Stampanoni-Panariello, B. Hemmerling, W. Hubschmid, Phys. Rev. A 51, 655 (1995)

7. M. Neracher, W. Hubschmid, Appl. Phys. B 79, 783 (2004)

8. H.J. Eichler, P. Günther, D.W. Pohl, Laser-Induced Dynamic Gratings (Springer, Berlin, 1986)

9. R.W. Boyd, Nonlinear Optics (Academic Press, New York, 1992)

10. Encyclopédie des gaz (Elsevier, Amsterdam, 1976)

11. S. Petralia, Nuovo Cimento 1, 351 (1955) 